\title{
Anomalous conductance scaling in strained Weyl semimetals
}

\author{
Jan Behrends $\odot,,^{1,2}$ Roni Ilan $\odot,{ }^{3}$ and Jens H. Bardarson $\oplus^{4}$ \\ ${ }^{1}$ Max-Planck-Institut für Physik komplexer Systeme, 01187 Dresden, Germany \\ ${ }^{2}$ T.C.M. Group, Cavendish Laboratory, University of Cambridge, J.J. Thomson Avenue, Cambridge CB3 OHE, United Kingdom \\ ${ }^{3}$ Raymond and Beverly Sackler School of Physics and Astronomy, Tel-Aviv University, Tel-Aviv 69978, Israel \\ ${ }^{4}$ Department of Physics, KTH Royal Institute of Technology, Stockholm SE-106 91, Sweden
}

(Received 24 June 2019; published 27 November 2019)

\begin{abstract}
Magnetotransport provides key experimental signatures in Weyl semimetals. The longitudinal magnetoresistance is linked to the chiral anomaly and the transversal magnetoresistance to the dominant charge relaxation mechanism. Axial magnetic fields that act with opposite sign on opposite chiralities facilitate new transport experiments that probe the low-energy Weyl nodes. As recently realized, these axial fields can be achieved by straining samples or adding inhomogeneities to them. Here, we identify a robust signature of axial magnetic fields: an anomalous scaling of the conductance in the diffusive ultraquantum regime. In particular, we demonstrate that the longitudinal conductivity in the ultraquantum regime of a disordered Weyl semimetal subjected to an axial magnetic field increases with both the field strength and sample width due to a spatial separation of charge carriers. We contrast axial magnetic with real magnetic fields to clearly distinguish the different behavior of the conductance. Our results rely on numerical tight-binding simulations and are supported by analytical arguments. We argue that the spatial separation of charge carriers can be used for directed currents in microstructured electronic devices.
\end{abstract}

DOI: 10.1103/PhysRevResearch.1.032028

Small variations in the parameters of a Hamiltonian describing a solid can affect the low-energy degrees of freedom in the form of a pseudofield, mimicking the effects of an external field. Such pseudofields can have properties that are fundamentally different from the fields commonly realized in nature. Inhomogeneities in Weyl semimetals [1-4], for example, effectively induce [5] axial pseudoelectric and pseudomagnetic fields [6-9], similar to strain-induced pseudofields in graphene [10-12]. These axial fields act with opposite sign on left- and right-handed Weyl fermions, which constitute the low-energy degrees of freedom in Weyl semimetals, enriching their electrodynamics by a broader set of fields [7,13-15]. As a consequence of axial magnetic fields, pseudo-Landau levels emerge at low energies $[8,9]$ while axial electric fields induce chirality-dependent transport $[16,17]$. Axial magnetic fields are not unique to Weyl semimetals but may also be be simulated in cold atom systems [18], and were demonstrated in various Weyl metamaterials $[19,20]$, but no definite manifestation has been found in the solid state.

Robust transport signatures for axial magnetic fields are still lacking. Although predicted to enhance the conductivity of a Weyl semimetal by additional anomaly-induced contributions [8,9], akin to effects of external magnetic fields [21-24], this enhancement relies on a local redistribution of charge [14], which leads to screening effects that may spoil

Published by the American Physical Society under the terms of the Creative Commons Attribution 4.0 International license. Further distribution of this work must maintain attribution to the author(s) and the published article's title, journal citation, and DOI. this contribution. In this work, we identify an anomalous scaling of the conductance in the ultraquantum limit as a unique signature of axial magnetic fields. This anomalous scaling, present for moderate disorder strengths, is induced by a spatial separation of counterpropagating modes, as we explain in the following. This is fundamentally different from transport in the presence of a regular magnetic field, which in the ultraquantum limit is dominated by chiral modes that are separated in momentum space but overlapping in real space.

In the following, we focus on the simplest time-reversalbreaking Weyl semimetals with only a single pair of Weyl nodes. The momentum-space separation $2 \mathbf{b}$ between the two Weyl nodes can at low energies be effectively regarded as a vector potential that acts with an opposite sign on these two Weyl nodes, a so-called axial vector potential. Inhomogeneities, e.g., strain-induced variations in the hopping amplitudes, may result in a spatial variation, $\mathbf{b} \rightarrow \mathbf{b}(\mathbf{r})[8,9]$. Generally, a spatially varying $\mathbf{b}$ gives rise to an axial magnetic field via [25]

$$
\mathbf{B}_{5}=\nabla \times \mathbf{b} .
$$

In a lattice, $\mathbf{b}$ must go to zero at the boundaries of an open system [9]; for periodic boundary condition, it must respect the periodicity [14]. Thus, any axial magnetic field $\mathbf{B}_{5}$ averages to zero [9], and any equilibrium current induced by $\mathbf{B}_{5}$ vanishes when integrated over the whole sample $[26,27]$.

The consequences of axial magnetic fields on charge transport can be intuitively understood by comparing the dispersion in the presence of magnetic and axial magnetic fields for a pair of Weyl nodes separated by a constant component along $z$, shown in Fig. 1. For concreteness we adopt a slab geometry and for the real magnetic field $\mathbf{B}=B \hat{z}$ we work in the Landau gauge $\mathbf{A}=B x \hat{y}$, which leaves $k_{y}$ as a good 


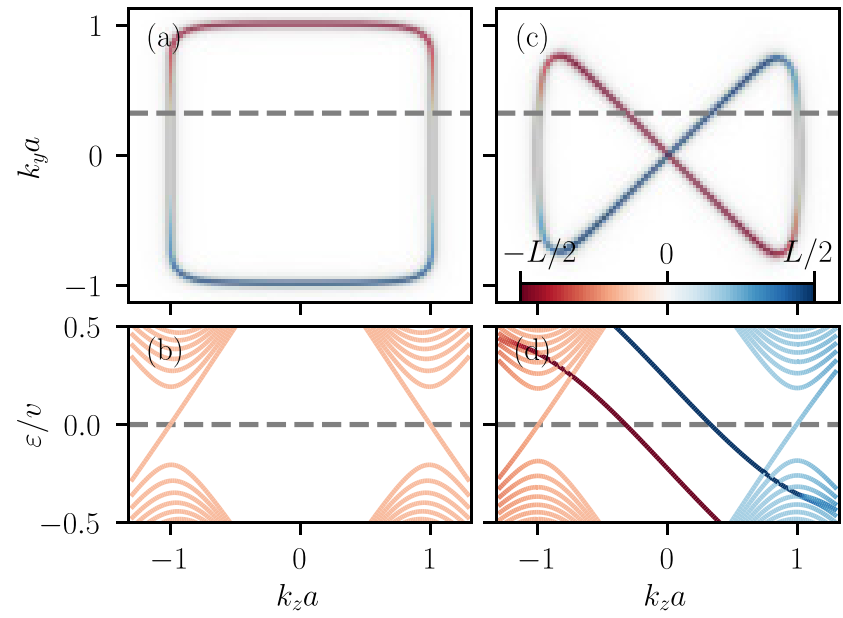

FIG. 1. Fermi surface and dispersion of a Weyl semimetal, Eq. (2), subjected to a magnetic field $\mathbf{B}$, panels (a) and (b), and to an axial magnetic field $\mathbf{B}_{5}$, panels (c) and (d). The color denotes the real-space localization $\langle x\rangle$ of the states; cf. inset panel (c). We choose open boundary conditions with $L / a=100$ sites in the $x$ direction and a (pseudo-)magnetic length of $\ell_{B}=\ell_{5}=7.07 a$ with lattice constant $a$. (a) Fermi surface in presence of $\mathbf{B}=B \hat{z}$. (b) Corresponding dispersion at $k_{y}=0.19 / a$ [dashed lines in panels (a) and (c)]. The dashed lines in panels (b) and (d) show the Fermi energy $\varepsilon_{F}=0$ as a guide for the eyes. (c) Fermi surface in presence of bulk $\mathbf{B}_{5}=B_{5} \hat{z}$ that results in pseudo-Landau levels with opposite position-momentum locking. (d) Corresponding dispersion at $k_{y}=0.19 / a$.

quantum number. In the presence of a magnetic field, the bulk Landau levels are exponentially localized at $x=\ell_{B}^{2} k_{y}$ with localization length $\ell_{B}=\sqrt{\hbar /(e B)}$. At each surface, Fermi arcs connect the bulk Landau levels of opposite chirality at the same momentum $k_{y}=L /\left(2 \ell_{B}^{2}\right)$, resulting in a rectangular Fermi surface [Fig. 1(a)]. Figure 1(b) shows the energy dispersion at a constant $k_{y}$. The bulk zeroth Landau levels are counterpropagating and localized at the same real-space position due to position-momentum locking. Thus, backscattering is possible via scattering between these levels.

In the presence of a constant bulk $\mathbf{B}_{5}$, pseudo-Landau levels form. Since $\mathbf{B}_{5}$ acts with opposite sign on the two chiralities, the bulk Landau levels propagate in the same direction. Assuming the $\mathbf{B}_{5}$ is generated by a variation $\mathbf{b} \propto x \hat{y}$, these levels are exponentially localized at $x= \pm \ell_{5}^{2} k_{y}$, with the sign determined by the chirality and the pseudomagnetic length $\ell_{5}=1 / \sqrt{B_{5}}$. Surface states connect the bulk pseudo-Landau levels of opposite chiralities at different $k_{y}= \pm L /\left(2 \ell_{5}^{2}\right)$, such that surface states twist in momentum space [Fig. 1(c)]. In this example, with a constant component $b_{z} \hat{z}$ parallel to $\mathbf{B}_{5}$, the Fermi surface traces a bowtie [14]. A momentum-space cut at constant $k_{y}$ [Fig. 1(d)] reveals that the bulk pseudo-Landau levels propagate in the same direction. Thus, scattering between those states does not contribute to the transport time. Since $\mathbf{B}_{5}$ averages to zero over the whole sample, surface states propagate in the opposite direction. Accordingly, only scattering processes between the counterpropagating bulk and surface contribute to the transport time. Charges localized deep in the bulk must scatter all the way to the surface to relax.

As a consequence of the limitation of backscattering to bulk-to-surface scattering, we predict a robust experimental signature of an axial magnetic field observable in simple transport measurements: the scaling of conductance with the system's width. We find that the conductance along $\mathbf{B}_{5}$ increases with the width cubed, different from the width squared, usually obtained in diffusive systems [28]. This anomalous scaling originates in the spatial separation of left- and rightmoving modes, with one confined to the bulk and the other to the surface, as argued in Ref. [8]. Because of the spatial separation, scattering mechanisms are substantially modified compared to standard magnetotransport experiments, as we elaborate on below. We explicitly demonstrate the scaling by employing tight-binding simulations, including the effects of disorder, to compute the conductance. We use the Hamiltonian

$$
\begin{aligned}
\mathcal{H}_{\mathbf{k}}= & v\left[\sin \left(k_{y} a\right) \sigma_{x}-\sin \left(k_{x} a\right) \sigma_{y}\right] \tau_{z}+v \sin \left(k_{z} a\right) \tau_{y} \\
& +t \sum_{i}\left[1-\cos \left(k_{i} a\right)\right] \tau_{x}+v a \mathbf{u} \cdot \mathbf{b} .
\end{aligned}
$$

with the lattice constant $a$, and $\mathbf{u}=\left(-\sigma_{x} \tau_{x},-\sigma_{y} \tau_{x}, \sigma_{z}\right)$. The Pauli matrices $\sigma_{\mu}$ and $\tau_{\mu}$ act on different degrees of freedom, e.g., spin and orbital. We discuss a time-reversal invariant model with four Weyl nodes, which allows for bulk-to-bulk backscattering, in Ref. [29]. When $t=2 v / \sqrt{3}$, as throughout this work, $2 \mathbf{b}$ equals the Weyl node separation in momentum space with deviations $O\left(\mathbf{b}^{5}\right)$ when b points along a primitive lattice vector, and deviations $O\left(\mathbf{b}^{3}\right)$ otherwise. We choose $\mathbf{b}=\left(0, B_{5} x, b_{z}\right)$ with $x \in\left[-L_{x} / 2, L_{x} / 2\right]$ to generate an axial magnetic field $\mathbf{B}_{5}=B_{5} \hat{z}$ parallel to the constant component $b_{z}$. We take open boundary conditions in the $x$ direction and average over twisted periodic boundary conditions in the $y$ direction $\psi\left(y+L_{y}\right)=e^{i \phi} \psi(y)$. The transport direction is $z$, parallel to $\mathbf{B}_{5}$ and $\mathbf{B}$.

We perform all transport calculations using KWANT [30]. The total system consists of a sample of length $L_{z}=L_{\|}$in the $z$ direction and width $L_{x}=L_{y}=L_{\perp}$ in both $x, y$ directions. It is connected to two semi-infinite clean leads governed by the same Hamiltonian (2) at the chemical potential $\mu_{\text {lead }}$ with the same node separation and (axial) magnetic field.

For clean systems and $\mu_{\text {lead }}=0$, transport is ballistic, giving the conductance $G=n e^{2} / h$ for $n$ propagating modes. For both $\mathbf{B}$ and $\mathbf{B}_{5}, n$ increases linearly with the sample's cross section $L_{\perp}^{2}$ and the field strength. For $\mathbf{B}$, the number of propagating modes equals the degeneracy of the bulk Landau levels, and for $\mathbf{B}_{5}, n$ equals twice the degeneracy since surface states contribute equally to transport. The Landau level degeneracy increases linearly with $L_{\perp}^{2}$ and $B\left(B_{5}\right)$. Thus, for a clean system, the scaling of the conductance with $L_{\perp}^{2}$ and the field strength is the same for both $B$ and $B_{5}$.

In Figs. 2(a) and 2(b), we show the conductance as a function of $B$ and $B_{5}$, respectively, which indeed increases linearly with the field strength. In the presence of $B_{5}$, the linear regime breaks down at a field strength characterized by $B_{5} \propto a^{2} / \ell_{5}^{2} \gtrsim 0.1$. This breakdown is a lattice effect: $\mathbf{b}$ equals the Weyl node separation only when neglecting higher order corrections; the equality breaks down for large $|\mathbf{b}| \gg 1 / a$, i.e., when $B_{5} L_{\perp} \gg 1 / a$. Then, the conductance increases faster with $B_{5}$ [Fig. 2(b)]. To avoid this lattice-induced effect, we limit the size of both $B_{5}$ and $L_{\perp}$ in the subsequent discussion. Furthermore, we observe that the ratio of the conductance in the presence of $\mathbf{B}_{5}$ and $\mathbf{B}$ is smaller than 2; cf. Fig. 2(c). This lattice effect only affects the total conductance in the 


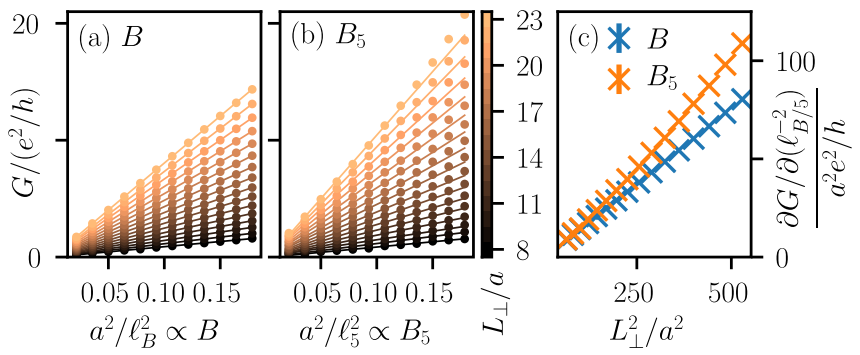

FIG. 2. Conductance of clean samples in the presence of (axial) magnetic fields. (a) Numerically obtained conductance (circles) as a function of the field strength $B\left(\mathbf{B}_{5}=0\right)$ for transport along $\hat{z} \| \mathbf{B}$. The conductance is independent of $L_{\|}$and grows linearly with $B$ (linear fit indicated by solid lines). (b) Similarly, the conductance grows linearly with $B_{5}(\mathbf{B}=0)$ up to $a^{2} / \ell_{5}^{2} \lesssim 0.12$. (c) The slope of $\partial G / \partial B_{(5)}$ increases linearly with $L_{\perp}^{2}$, such that $G \propto B L_{\perp}^{2}$ $\left(G \propto B_{5} L_{\perp}^{2}\right)$. The ratio of the slopes in panel (c) is 1.29.

presence of $B_{5}$, but not its scaling with the field strength or cross section, so we neglect it in our following analysis.

To discuss (axial) longitudinal magnetotransport in the presence of disorder, we add both vector and scalar disorder by introducing a random on-site matrix $V\left(\mathbf{r}_{i}\right)$ satisfying

$$
\begin{gathered}
V\left(\mathbf{r}_{i}\right)=\sum_{\mu \nu} v_{\mu \nu}\left(\mathbf{r}_{i}\right) \sigma_{\mu} \tau_{\nu}, \\
\left\langle\left\langle v_{\mu \nu}\left(\mathbf{r}_{i}\right) v_{\rho \sigma}\left(\mathbf{r}_{j}\right)\right\rangle\right\rangle=g_{\mu \nu} K\left(\mathbf{r}_{i}-\mathbf{r}_{j}\right) \delta_{\mu \rho} \delta_{\nu \sigma}
\end{gathered}
$$

to the lattice Hamiltonian. The function $K\left(\mathbf{r}_{i}-\mathbf{r}_{j}\right)$ is the disorder correlator. Since the presence of Weyl nodes in the Hamiltonian (2) does not rely on any symmetries, no restrictions are put on the matrix structure of disorder and we set for convenience $g_{\mu \nu}=1$.

For a better comparison between real and axial magnetic fields, we briefly review previous work on the magnetoconductivity. For systems larger than the mean free path, transport is diffusive and the conductance scales as $G=\sigma L_{\perp}^{2} / L_{\|}$with a conductivity $\sigma$ independent of the system's dimensions [28]. The scaling of $\sigma$ with $B$ depends on the disorder type. For white-noise disorder

$$
K\left(\mathbf{r}_{i}-\mathbf{r}_{j}\right)=\frac{W^{2}}{12} \delta_{i j},
$$

the conductivity in the ultraquantum limit is independent of the magnetic field $[31,32]$. Gaussian correlations in the disorder potential with correlation length $\xi$ change the conductivity scaling to $\sigma \propto\left(\xi^{2}+\ell_{B}^{2}\right) / \ell_{B}^{2}$ [33-35]. In the strong-field limit $\ell_{B} \ll \xi, \sigma$ increases with $\ell_{B}^{-2}$, i.e., with the magnetic field [29]. When $\ell_{B} \gg \xi$, the scaling for white-noise disorder is recovered, consistent with observations in TaAs [36].

Replacing the magnetic field by an axial magnetic field results in two changes: First, the conductivity increases linearly with $B_{5}$ for white-noise disorder and quadratically with $B_{5}$ for Gaussian-correlated disorder in the strong-field limit [8]. Second, as argued above, the conductivity increases with the system's width due to the spatial separation of counterpropagating modes. As soon as disorder becomes large enough to mix the zeroth and higher Landau levels, all effects dominated by the zeroth Landau levels start to wash out-especially phenomena driven by the copropagating bulk zeroth Landau levels for $\mathbf{B}_{5}$ and counterpropagating Landau levels for $\mathbf{B}$. We thus expect that the conductance scales similarly with field strength and system dimensions for axial and magnetic fields in strongly disordered systems.

To account for the scaling of the conductivity with the system's width, we define the dimensionless conductivity $g\left(L_{\perp}\right)$, a quantity not independent of the system's dimensions, via

$$
G=\frac{e^{2}}{h a} \frac{L_{\perp}^{2}}{L_{\|}} g\left(L_{\perp}\right) .
$$

We consider the regime where $g\left(L_{\perp}\right)$ is independent of $L_{\|}$, i.e., the diffusive regime where $G \propto 1 / L_{\|}$.

To access the diffusive regime in tight-binding simulations, we need to avoid the ballistic and localized limits. When $L_{\|} \gg L_{\perp}$, the system is essentially one dimensional and the charge carriers always localize, giving an exponentially decreasing conductance $[37,38]$. If the mean free path is larger than the system size, transport is ballistic, observable for $W / v \lesssim 1.5$ and the system sizes we consider. To access the regime where the (axial) magnetic field influences transport, the (pseudo-)magnetic length [that sets the localization length of the (pseudo-)Landau levels] must be smaller than the sample thickness. In our numerical simulations, the last restriction results in magnetic lengths ranging from $\ell_{B}=2.6 a$ to $\ell_{B}=4.8 a$, which require large (axial) magnetic fields; however, in mesoscopic samples, the condition $\ell_{B} / L \ll 1$ is much more easily satisfied than in the numerical simulations; i.e., only intermediate field strengths are necessary.

We numerically investigate the conductance as a function of $L_{\|}$in the presence of white-noise disorder (5). We focus on the regime where the inverse conductance increases linearly with $L_{\|}$, which we can observe up to $L_{\|} \lesssim 3 L_{\perp}$ before reaching the one-dimensional limit showing onset of localization. In Fig. 3, we compare transport in the presence of axial fields with magnetic fields for different field strengths characterized by its (pseudo-)magnetic length. Figures 3(a) and 3(b) show the conductance as a function of $L_{\|}$, normalized by the cross section $L_{\perp}^{2}$. We find that the dimensionless conductivity (6), proportional to the inverse of the slope $\partial G^{-1} / \partial L_{\|}$, does not increase with $B$ for sufficiently large disorder $W \gtrsim 2.5 v$ [Fig. 3(c)] as expected from $\tau_{v} \propto 1 / B$ for white-noise disorder $[33,35]$. In contrast, $g$ increases approximately linearly with the strength of the axial field $B_{5}$, Fig. 3(d). The slope $\partial g / \partial B_{5}$ decreases with the disorder strength $W$ before it eventually reaches zero; cf. inset in Fig. 3(c). When the disorder is larger than the spacing of the Landau levels $\left(W / v>a / \ell_{B}\right.$ or $\left.W / v>a / \ell_{5}\right)$, higher Landau levels start to contribute to transport, allowing bulk-to-bulk backscattering, gradually decreasing the difference between $B$ and $B_{5}$ in the transport signature.

To contribute to transport relaxation, charges need to scatter the distance $L_{\perp}$, which takes $\left(L_{\perp} / \ell_{5}\right)^{2} \propto B_{5}$ scattering events [8]. The scaling of the conductivity $g \propto B_{5}$ we observe is different from the prediction in Ref. [8], since in our case $\tau_{v} \propto 1 / B_{5}$ due to white-noise disorder. For completeness, we analytically compute the scattering amplitude to lowest order in disorder in Ref. [29] and show that it decreases faster with system size than the amplitude from multiple scattering events.

We show the scaling of the conductivity as a function of $L_{\perp}$ in Fig. 4. As evident from Figs. 4(a) and 4(b), the inverse 


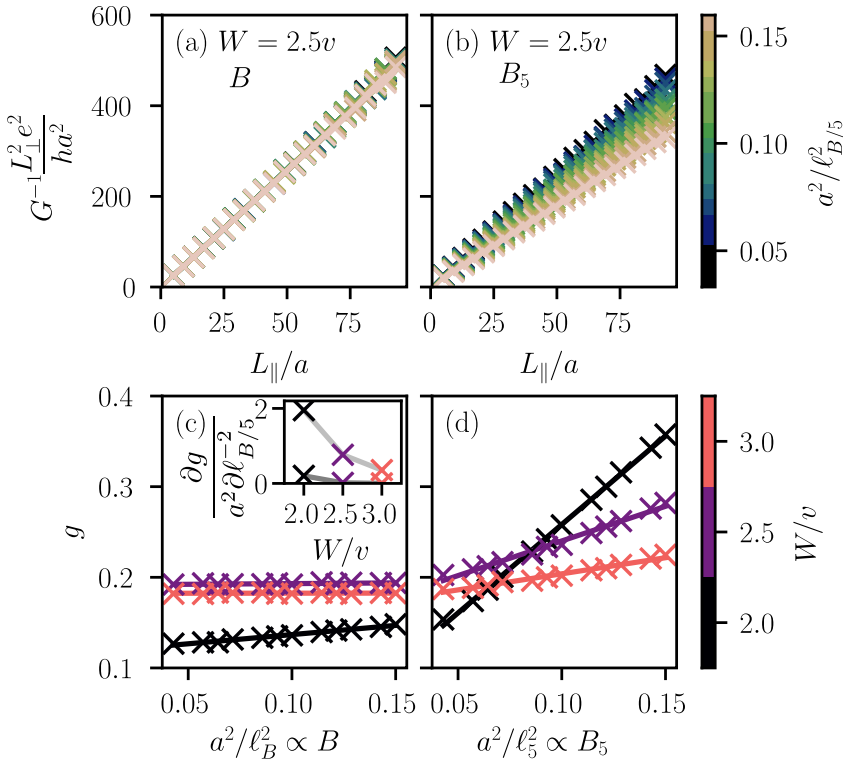

FIG. 3. Numerically obtained inverse conductance (a) in presence of $\mathbf{B}$ and (b) in presence of $\mathbf{B}_{5}$ as a function of $L_{\|}$, the system size in transport direction. The colors denote different values of the (pseudo-)magnetic length $\ell_{B}\left(\ell_{5}\right)$. The slope from panels (a) and (b) is the inverse of the dimensionless conductivity $g$, shown in panels (c) and (d) as a function of the (axial) magnetic field strength for different disorder strengths $W / v$. While $g$ clearly increases with $B_{5}$, it stays almost constant in presence of $B$ and disorder $W / v \gtrsim 2.5$. The inset in panel (c) shows the slope $\partial g / \partial L_{\perp}$ for different disorder strengths and $B$ (dark gray) and $B_{5}$ (light gray). All results are averaged over twisted boundary conditions and 100 disorder configurations; the chemical potential in the leads is $\mu_{\text {lead }}=1.5 v$, and the transversal width $L_{\perp}=21 a$.

conductance $G^{-1}$ increases linearly with $L_{\|}$when $L_{\|} \lesssim 3 L_{\perp}$ for both $B$ and $B_{5}$ and various values of $L_{\perp}$. The dimensionless conductivity (6) is independent of $L_{\perp}$ in the presence of $B$, Fig. 4(c), but increases approximately linearly with $L_{\perp}$ in the presence of $B_{5}$, Fig. 4(d). This is one of our main results: The conductivity increases linearly with the sample's thickness in the ultraquantum regime when $L_{\perp} \gg \ell_{5}$, corresponding to a conductance that increases with the thickness cubed, which we observe numerically for moderately large disorder $(1.5 \lesssim W / v \lesssim 3.5)$. The linear scaling of the conductivity with $L_{\perp}$ arises from subleading contributions in $L_{\perp}$ to the scattering time [29]. Similar to the scaling with $B_{5}$, the slope with $L_{\perp}$ decreases with the disorder strength, due to the mixing with higher Landau levels [inset in Fig. 4(c)]. A dependence of the conductivity on a systems thickness is a known indicator for surface effects. Indeed, in our case, the only effective transport relaxation is scattering between bulk and surface modes. As the thickness grows, more modes localized deep in the bulk become less affected by such scattering, and the conductivity is enhanced.

To conclude, in this work, we numerically investigated how charge transport in Weyl semimetals is affected by the presence of axial magnetic fields and white-noise disorder. We explicitly demonstrated that the conductivity increases with both $B_{5}$, the strength of the axial field, and $L_{\perp}$, the transverse width of the system. Both observations can be intuitively
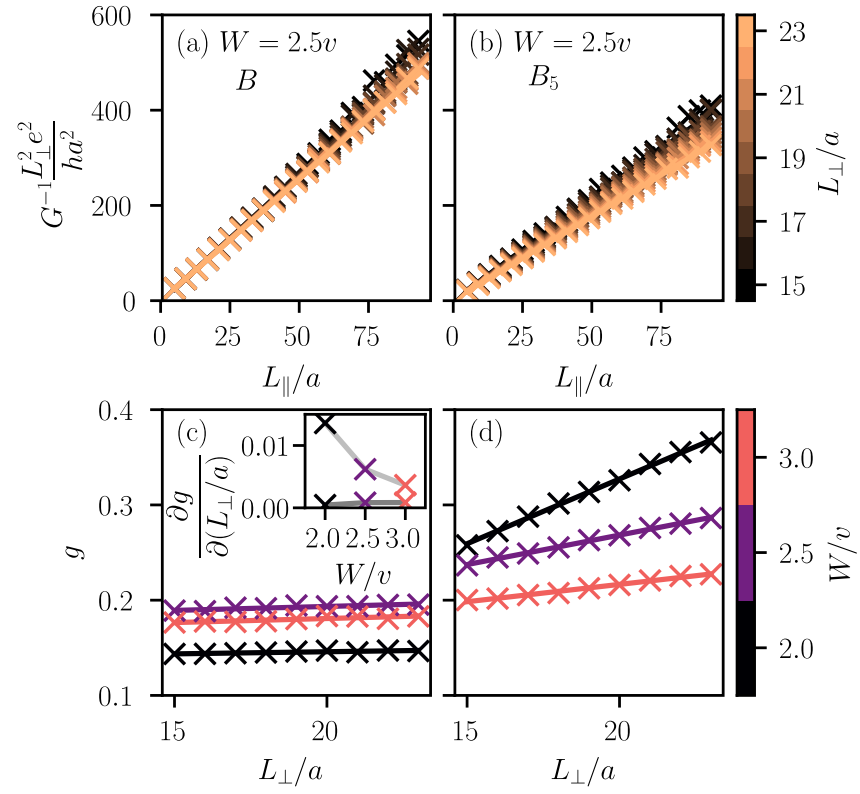

FIG. 4. Numerically obtained inverse conductance (a) in the presence of $\mathbf{B}$ and (b) in the presence of $\mathbf{B}_{5}$ as a function of $L_{\|}$. The colors denote different values of $L_{\perp}$. The slope from panels (a) and (b) is the inverse of the dimensionless conductivity $g$, shown in panels (c) and (d) as a function of $L_{\perp}$ for different disorder strengths $W / v$. While $g$ increases linearly with $L_{\perp}$ in the presence of axial fields, it stays, apart from finite-size effects, constant in presence of magnetic fields $B$, as evident from the inset in panel (c) that shows the slope $\partial g / \partial L_{\perp}$ for $B$ (dark gray) and $B_{5}$ (light gray). (Pseudo-)magnetic length $\ell_{B}=2.65 a\left(\ell_{5}=2.65 a\right)$; other parameters as in Fig. 3 .

understood by the real-space separation of counterpropagating modes in the presence of $B_{5}$.

We are confident that the conductance scaling uncovered in this work can be observed in experiments, considering recent efforts in manufacturing microstructured Weyl semimetals [39], creating strain-induced fields in type-II Weyl semimetals [40], and transport experiments in Dirac nanowires [41,42]. As our results rely on time-reversal symmetry-breaking materials, magnetic-exchange-induced Weyl semimetals $[43,44]$ are promising platforms for experiments. The conditions necessary to observe the unusual scaling of the conductivity are sample width larger than the magnetic length, and sample length in the transport direction larger than the mean free path. Our results show that the spatial separation of leftand right-moving charge carriers [8] is stable toward small disorder, which can be used in devices that require a spatial separation of counterpropagating currents. We stress that the implications for these observations are far reaching as they imply that experimentally approaching the ultraquantum limit of Weyl semimetals does not require the generation of large magnetic fields [45] — moderate strain can easily induce axial field strengths of a few Tesla $[8,9,46]$.

We thank A. G. Grushin, D. A. Pesin, and D. I. Pikulin for insightful discussions. This work was supported by the ERC Starting Grant No. 678795 TopInSy and the ERC Starting Grant No. 679722. R.I. is supported by the ISF under Grant No. $1790 / 18$. 
[1] H. Weng, C. Fang, Z. Fang, B. A. Bernevig, and X. Dai, Weyl Semimetal Phase in Noncentrosymmetric TransitionMetal Monophosphides, Phys. Rev. X 5, 011029 (2015).

[2] S.-M. Huang, S.-Y. Xu, I. Belopolski, C.-C. Lee, G. Chang, B. Wang, N. Alidoust, G. Bian, M. Neupane, A. Bansil, H. Lin, and M. Z. Hasan, A Weyl Fermion semimetal with surface Fermi arcs in the transition metal monopnictide TaAs class, Nat. Commun. 6, 7373 (2015).

[3] S.-Y. Xu, I. Belopolski, N. Alidoust, M. Neupane, G. Bian, C. Zhang, R. Sankar, G. Chang, Z. Yuan, C.-C. Lee, S.-M. Huang, H. Zheng, J. Ma, D. S. Sanchez, B. Wang, A. Bansil, F. Chou, P. P. Shibayev, H. Lin, S. Jia, and M. Z. Hasan, Discovery of a Weyl Fermion semimetal and topological Fermi arcs, Science 349, 613 (2015).

[4] B. Q. Lv, H. M. Weng, B. B. Fu, X. P. Wang, H. Miao, J. Ma, P. Richard, X. C. Huang, L. X. Zhao, G. F. Chen, Z. Fang, X. Dai, T. Qian, and H. Ding, Experimental Discovery of Weyl Semimetal TaAs, Phys. Rev. X 5, 031013 (2015).

[5] Inhomogeneities additionally induce torsion and curvature to the underlying spacetime [47-51], which we neglect.

[6] M. N. Chernodub, A. Cortijo, A. G. Grushin, K. Landsteiner, and M. A. H. Vozmediano, Condensed matter realization of the axial magnetic effect, Phys. Rev. B 89, 081407(R) (2014).

[7] A. Cortijo, Y. Ferreirós, K. Landsteiner, and M. A. H. Vozmediano, Elastic Gauge Fields in Weyl Semimetals, Phys. Rev. Lett. 115, 177202 (2015).

[8] D. I. Pikulin, A. Chen, and M. Franz, Chiral Anomaly from Strain-Induced Gauge Fields in Dirac and Weyl Semimetals, Phys. Rev. X 6, 041021 (2016).

[9] A. G. Grushin, J. W. F. Venderbos, A. Vishwanath, and R. Ilan, Inhomogeneous Weyl and Dirac Semimetals: Transport in Axial Magnetic Fields and Fermi Arc Surface States from PseudoLandau Levels, Phys. Rev. X 6, 041046 (2016).

[10] J. L. Mañes, Symmetry-based approach to electron-phonon interactions in graphene, Phys. Rev. B 76, 045430 (2007).

[11] F. Guinea, M. I. Katsnelson, and A. K. Geim, Energy gaps and a zero-field quantum hall effect in graphene by strain engineering, Nat. Phys. 6, 30 (2010).

[12] N. Levy, S. A. Burke, K. L. Meaker, M. Panlasigui, A. Zettl, F. Guinea, A. H. C. Neto, and M. F. Crommie, Strain-induced pseudo-magnetic fields greater than 300 Tesla in graphene nanobubbles, Science 329, 544 (2010).

[13] K. Landsteiner, Notes on anomaly induced transport, Acta Phys. Pol. B 47, 2617 (2016).

[14] J. Behrends, S. Roy, M. H. Kolodrubetz, J. H. Bardarson, and A. G. Grushin, Landau levels, Bardeen polynomials, and Fermi arcs in Weyl semimetals: Lattice-based approach to the chiral anomaly, Phys. Rev. B 99, 140201(R) (2019).

[15] R. Ilan, A. G. Grushin, and D. I. Pikulin, Pseudo-electromagnetic fields in topological semimetals, arXiv:1903.11088.

[16] E. V. Gorbar, V. A. Miransky, I. A. Shovkovy, and P. O. Sukhachov, Pseudomagnetic lens as a valley and chirality splitter in Dirac and Weyl materials, Phys. Rev. B 95, 241114(R) (2017).

[17] E. V. Gorbar, V. A. Miransky, I. A. Shovkovy, and P. O. Sukhachov, Origin of Bardeen-Zumino current in lattice models of Weyl semimetals, Phys. Rev. B 96, 085130 (2017).

[18] S. Roy, M. Kolodrubetz, N. Goldman, and A. G. Grushin, Tunable axial gauge fields in engineered Weyl semimetals:
Semiclassical analysis and optical lattice implementations, 2D Mater. 5, 024001 (2018).

[19] V. Peri, M. Serra-Garcia, R. Ilan, and S. D. Huber, Axial-fieldinduced chiral channels in an acoustic Weyl system, Nat. Phys. 15, 357 (2019).

[20] H. Jia, R. Zhang, W. Gao, Q. Guo, B. Yang, J. Hu, Y. Bi, Y. Xiang, C. Liu, and S. Zhang, Observation of chiral zero mode in inhomogeneous three-dimensional Weyl metamaterials, Science 363, 148 (2019).

[21] D. T. Son and B. Z. Spivak, Chiral anomaly and classical negative magnetoresistance of Weyl metals, Phys. Rev. B 88, 104412 (2013).

[22] A. A. Burkov, Chiral anomaly and transport in Weyl metals, J. Phys.: Condens. Matter 27, 113201 (2015).

[23] Y. Zhang, D. Bulmash, P. Hosur, A. C. Potter, and A. Vishwanath, Quantum oscillations from generic surface Fermi arcs and bulk chiral modes in Weyl semimetals, Sci. Rep. 6, 23741 (2016).

[24] N. P. Armitage, E. J. Mele, and A. Vishwanath, Weyl and Dirac semimetals in three-dimensional solids, Rev. Mod. Phys. 90, 015001 (2018).

[25] R. A. Bertlmann, Anomalies in Quantum Field Theory, International Series of Monographs on Physics (Oxford University Press, Oxford, UK, 2000)

[26] H. Sumiyoshi and S. Fujimoto, Torsional Chiral Magnetic Effect in a Weyl Semimetal With a Topological Defect, Phys. Rev. Lett. 116, 166601 (2016).

[27] K. Kodama and Y. Takane, Persistent current due to a screw dislocation in Weyl semimetals: Role of one-dimensional chiral states, J. Phys. Soc. Jpn. 88, 054715 (2019).

[28] N. W. Ashcroft and N. D. Mermin, Solid State Physics (Cengage Learning, Andover, UK, 2011).

[29] See Supplemental Material at http://link.aps.org/supplemental/ 10.1103/PhysRevResearch.1.032028, which includes Refs. [52-62], for the discussion of a time-reversal symmetric model, numerical simulations of transport in presence of correlated disorder, and the analytical computation of the bulk-to-surface scattering rate.

[30] C. W. Groth, M. Wimmer, A. R. Akhmerov, and X. Waintal, KWANT: A software package for quantum transport, New J. Phys. 16, 063065 (2014).

[31] B. Z. Spivak and A. V. Andreev, Magnetotransport phenomena related to the chiral anomaly in Weyl semimetals, Phys. Rev. B 93, 085107 (2016).

[32] H.-Z. Lu and S.-Q. Shen, Quantum transport in topological semimetals under magnetic fields, Front. Phys. 12, 127201 (2017).

[33] H. Z. Lu, S. B. Zhang, and S. Q. Shen, High-field magnetoconductivity of topological semimetals with short-range potential, Phys. Rev. B 92, 045203 (2015).

[34] C. Z. Chen, H. Liu, H. Jiang, and X. C. Xie, Positive magnetoconductivity of Weyl semimetals in the ultraquantum limit, Phys. Rev. B 93, 165420 (2016).

[35] S. B. Zhang, H. Z. Lu, and S. Q. Shen, Linear magnetoconductivity in an intrinsic topological Weyl semimetal, New J. Phys. 18, 053039 (2016).

[36] B. J. Ramshaw, K. A. Modic, A. Shekhter, Y. Zhang, E.-A. Kim, P. J. W. Moll, M. D. Bachmann, M. K. Chan, J. B. Betts, F. Balakirev, A. Migliori, N. J. Ghimire, E. D. Bauer, F. Ronning, and R. D. McDonald, Quantum limit transport and 
destruction of the Weyl nodes in TaAs, Nat. Commun. 9, 2217 (2018).

[37] P. W. Anderson, Absence of diffusion in certain random lattices, Phys. Rev. 109, 1492 (1958).

[38] E. Abrahams, P. W. Anderson, D. C. Licciardello, and T. V. Ramakrishnan, Scaling Theory of Localization: Absence of Quantum Diffusion in Two Dimensions, Phys. Rev. Lett. 42, 673 (1979)

[39] M. D. Bachmann, N. Nair, F. Flicker, R. Ilan, T. Meng, N. J. Ghimire, E. D. Bauer, F. Ronning, J. G. Analytis, and P. J. Moll, Inducing superconductivity in Weyl semimetal microstructures by selective ion sputtering, Sci. Adv. 3, e1602983 (2017).

[40] S. Kamboj, P. S. Rana, A. Sirohi, A. Vasdev, M. Mandal, S. Marik, R. P. Singh, T. Das, and G. Sheet, Generation of strain-induced pseudo-magnetic field in a doped type-II Weyl semimetal, Phys. Rev. B 100, 115105 (2019).

[41] C.-Z. Li, L.-X. Wang, H. Liu, J. Wang, Z.-M. Liao, and D.-P. $\mathrm{Yu}$, Giant negative magnetoresistance induced by the chiral anomaly in individual $\mathrm{Cd}_{3} \mathrm{As}_{2}$ nanowires, Nat. Commun. 6, 10137 (2015).

[42] L.-X. Wang, S. Wang, J.-G. Li, C.-Z. Li, J. Xu, D. Yu, and Z.-M. Liao, Magnetotransport properties near the Dirac point of Dirac semimetal $\mathrm{Cd}_{3} \mathrm{As}_{2}$ nanowires, J. Phys.: Condens. Matter 29, 044003 (2017).

[43] J.-R. Soh, F. de Juan, M. G. Vergniory, N. B. M. Schröter, M. C. Rahn, D. Y. Yan, J. Jiang, M. Bristow, P. A. Reiss, J. N. Blandy, Y. F. Guo, Y. G. Shi, T. K. Kim, A. McCollam, S. H. Simon, Y. Chen, A. I. Coldea, and A. T. Boothroyd, Ideal Weyl semimetal induced by magnetic exchange, Phys. Rev. B 100, 201102(R) (2019).

[44] H. Su, B. Gong, W. Shi, H. Yang, H. Wang, W. Xia, Z. Yu, P.-J. Guo, J. Wang, L. Ding, L. Xu, X. Li, X. Wang, Z. Zou, N. Yu, Z. Zhu, Y. Chen, Z. Liu, K. Liu, G. Li, and Y. Guo, Magnetic exchange induced Weyl state in a semimetal $\mathrm{EuCd}_{2} \mathrm{Sb}_{2}$, arXiv:1903.12532.

[45] The ultraquantum limit requires large fields such that the chemical potential $\mu<\hbar v / \ell_{B}$, corresponding to fields $B>$ $\mu^{2} /\left(\hbar e v^{2}\right)$. For a Fermi velocity $v=10^{6} \mathrm{~m} / \mathrm{s}$ and $\mu=50 \mathrm{meV}$, fields of at least $B=4 \mathrm{~T}$ are necessary.

[46] T. Liu, D. I. Pikulin, and M. Franz, Quantum oscillations without magnetic field, Phys. Rev. B 95, 041201(R) (2017).

[47] L. Alvarez-Gaumé and E. Witten, Gravitational anomalies, Nucl. Phys. B 234, 269 (1984).

[48] O. Parrikar, T. L. Hughes, and R. G. Leigh, Torsion, parity-odd response, and anomalies in topological states, Phys. Rev. D 90, 105004 (2014).

[49] A. Cortijo and M. A. Zubkov, Emergent gravity in the cubic tight-binding model of Weyl semimetal in the presence of elastic deformations, Ann. Phys. (NY) 366, 45 (2016).
[50] A. Westström and T. Ojanen, Designer Curved-Space Geometry for Relativistic Fermions in Weyl Metamaterials, Phys. Rev. X 7, 041026 (2017).

[51] Y. Ferreiros, Y. Kedem, E. J. Bergholtz, and J. H. Bardarson, Mixed Axial-Torsional Anomaly in Weyl Semimetals, Phys. Rev. Lett. 122, 056601 (2019).

[52] K. Y. Yang, Y. M. Lu, and Y. Ran, Quantum Hall effects in a Weyl semimetal: Possible application in pyrochlore iridates, Phys. Rev. B 84, 075129 (2011).

[53] E. V. Gorbar, V. A. Miransky, I. A. Shovkovy, and P. O. Sukhachov, Origin of dissipative Fermi arc transport in Weyl semimetals, Phys. Rev. B 93, 235127 (2016).

[54] J. Xiong, S. K. Kushwaha, T. Liang, J. W. Krizan, M. Hirschberger, W. Wang, R. J. Cava, and N. P. Ong, Evidence for the chiral anomaly in the Dirac semimetal Na3Bi, Science 350, 413 (2015).

[55] X. Huang, L. Zhao, Y. Long, P. Wang, D. Chen, Z. Yang, H. Liang, M. Xue, H. Weng, Z. Fang, X. Dai, and G. Chen, Observation of the Chiral-Anomaly-Induced Negative Magnetoresistance: In 3D Weyl Semimetal TaAs, Phys. Rev. X 5, 031023 (2015).

[56] B. Shen, X. Deng, G. Kotliar, and N. Ni, Fermi surface topology and negative magnetoresistance observed in centrosymmetric $\mathrm{NbAs}_{2}$ semimetal, Phys. Rev.B 93, 195119 (2016).

[57] Y. Li, L. Li, J. Wang, T. Wang, X. Xu, C. Xi, C. Cao, and J. Dai, Resistivity plateau and negative magnetoresistance in the topological semimetal $\mathrm{TaSb}_{2}$, Phys. Rev. B 94, 121115(R) (2016).

[58] H. Li, H. He, H.-Z. Lu, H. Zhang, H. Liu, R. Ma, Z. Fan, S.-Q. Shen, and J. Wang, Negative magnetoresistance in dirac semimetal $\mathrm{Cd}_{3} \mathrm{As}_{2}$, Nat. Commun. 7, 10301 (2016).

[59] F. Arnold, C. Shekhar, S.-C. Wu, Y. Sun, R. D. dos Reis, N. Kumar, M. Naumann, M. O. Ajeesh, M. Schmidt, A. G. Grushin, J. H. Bardarson, M. Baenitz, D. Sokolov, H. Borrmann, M. Nicklas, C. Felser, E. Hassinger, and B. Yan, Negative magnetoresistance without well-defined chirality in the Weyl semimetal TaP, Nat. Commun. 7, 11615 (2016).

[60] R. D. D. Reis, M. O. Ajeesh, N. Kumar, F. Arnold, C. Shekhar, M. Naumann, M. Schmidt, M. Nicklas, and E. Hassinger, On the search for the chiral anomaly in Weyl semimetals: The negative longitudinal magnetoresistance, New J. Phys. 18, 085006 (2016).

[61] J. Xiong, S. Kushwaha, J. Krizan, T. Liang, R. J. Cava, and N. P. Ong, Anomalous conductivity tensor in the Dirac semimetal $\mathrm{Na}_{3} \mathrm{Bi}$, Europhys. Lett. 114, 27002 (2016).

[62] J. Behrends and J. H. Bardarson, Strongly angle-dependent magnetoresistance in Weyl semimetals with long-range disorder, Phys. Rev. B 96, 060201(R) (2017). 